\title{
Fast Drift-Scan CCD Imaging and Photometry with Small Telescopes: Lunar Occultations and Speckle Interferometry
}

\author{
Jorge Núñez \& Octavi Fors \\ Departament d'Astronomia i Meteorologia, Universitat de Barcelona, \\ Av. Diagonal 647, E-08028 Barcelona and Observatori Fabra, \\ Barcelona, Spain. E-mail: jorge@am.ub.es
}

\begin{abstract}
In this paper we show how inherent features of typical CCD cameras can be used for fast (in the order of milliseconds) photometric observations (FPO), even with submeter class telescopes. This is based on a modified drift-scanning technique which we show to be advantageous for such kind of observations. In particular, we successfully carried out some lunar occultations and speckle interferometry observations using this technique. During the year 2000, we registered several occultation events. In particular, we present the occultation of SAO79031. The millisecond photometry of the event allows us subsequent data analysis for stellar diameter estimation and close binary detection. We also show an example of the application of the technique to speckle interferometry observations. Finally, we present the main characteristics of our project to build a new remotely controlled observatory near Barcelona.
\end{abstract}

\section{Introduction}

Since the appearance of CCDs more than two decades ago, their technical specifications have been constantly improved. Despite this rapid development, most current research grade cameras are still not able to meet the read out speed which FPO programs demand. These should be run in millisecond sampling regime with low read out noise and high digitization resolution mode. This is the main reason why CCDs have traditionally been discarded as eventual detectors in favor of photoelectric photometer systems (PEP).

With the advent of adaptive optics, a few state-of-art frame transfer CCDs (FTCCD) have been released to work as part of wavefront sensor systems (Ragazzoni et al. 1998). These devices reach $3 \mathrm{Mpixel} / \mathrm{sec}$ at moderate read out noise regime, meeting most of the FPO requirements. While it is certain that they will play a key role in near future FPO programs, their cost is still far from making them accesible to most of the professional and high-end amateur community. Moreover, FTCCDs small active area makes them be dedicated detectors, which cannot be used for general imaging purposes.

In this paper we will show how standard full frame CCDs can be successfully used for FPO when operating in a modified drift-scanning mode. 


\section{Drift-Scanning}

The conventional use of a CCD device is the operation in stare mode where the CCD chip is read out at the end of the exposure. Once the shutter is closed, the charge generated by the incident light on the surface of the CCD is converted to digital numbers, on a line per line basis, as a clocked charge moves through a serial register. This has been the usual operating mode in astronomy for years.

However, other modes can be considered since the clocking rate $\tau$ can normally be specified by the user. Typically, one has three options for the $\tau$ value: $\tau=\tau_{0}, \tau<\tau_{0}$ or $\tau>\tau_{0}$, where $\tau_{0}$ is the sidereal rate at a given declination.

In the first case, acquired data appear as point-like sources provided clocking charge direction coincides with star motion over the chip and the telescope tracking system is disconnected. In the second case it is also necessary to have the camera properly aligned but, in this case, to ensure that the acquired data appear as point-like sources, it is necessary to slow the telescope tracking. These two variants in scanning mode are usually referred to as drift-scanning and time delay integration (TDI). This is the way several meridian circles (Stone et al. 1996) and Schmidt cameras (Sabbey, Coppi, \& Oemler 1998) observe for fast sky coverage at moderate limiting magnitude.

Regarding the third case, $\tau$ can be chosen according to the time scale and magnitude of the event to be recorded. The detector does not need to be specifically oriented, because we keep the telescope tracking all the time. Thus, the star remains stationary over the chip while photogenerated charge is clocked through the serial register at desired rate $\tau$. It is worth noting that a measure of the star flux is actually obtained every time a column is read out. In standard full frame CCDs this can be typically done at frequencies of 50 to $500 \mathrm{kHz}$, fast enough for most FPO. We see, therefore, how a non-dedicated detector such as a full frame CCD, very common among instrumental settings in astronomical observatories, could be used for recording fast photometry events.

\section{Application of Fast Drift-Scanning to Lunar Occultation Events}

Fast photometry of lunar occultations (LO), with a time resolution of one millisecond, allows us to obtain an angular resolution at milliarcsecond level of object features far beyond the seeing effect, allowing, for example, stellar angular diameter measurements and close binary detection. Telescope diameter (D) and filter bandwidth $(\Delta \lambda)$ are usually the two instrumental parameters that constrain the limiting angular resolution attainable by LO observations. However, this limit can be overcome with adequate data analysis if the lightcurve signal-to-noise ratio (SNR) is large enough.

Lunar occultation work has traditionally been performed using PEPs. These have been found to be extremely fast devices but show reduced quantum efficiency (QE 20\%) (Kristian \& Blouke 1982). On the other hand, CCDs with moderate-low read out noise would offer a better SNR due to their higher QE. Thus, as SNR plays a key role in data analysis, the selection of a detector with high QE and low read out noise turns out to be a crucial issue.

In this paper we examine the small-telescope regime for FPO. In particular, a $0.35 \mathrm{~m}$ Schmidt Cassegrain Telescope (Celestron-14) parallelally assembled to 


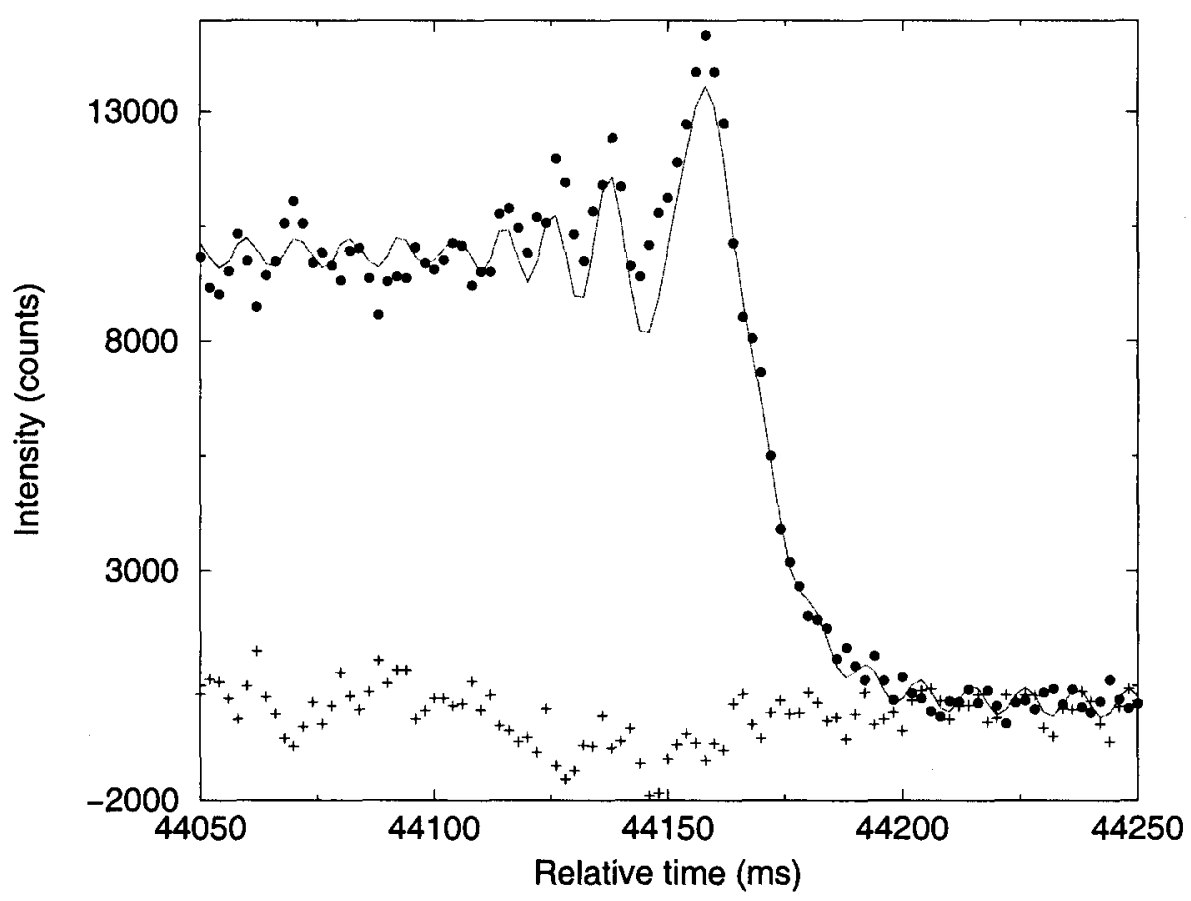

Figure 1. SAO79031 4.0mag $\left(4.5 \& 4.5 ; 0.1^{\prime \prime}\right)$ occultation at Fabra Observatory.

the Mailhat double $38 \mathrm{~cm}$-astrograph at Fabra Observatory was used. As detector, we employed a Texas Instruments TC-211 CCD (full frame chip, 192x164 $13.75 \times 16 \mu$ pixels, at $30 \mathrm{KHz}, \mathrm{QE} \sim 70 \%$ peak, 12e- rms). According to former specifications, TC-211 roughly suits FPO requirements.

Table 1. Data for SAO79031 occultation on 19:59:29 TU 03/14/2000.

\begin{tabular}{cccccccc}
\hline Object & Filter & $\lambda_{0} \pm \Delta \lambda(\mathrm{nm})$ & $\Delta \mathrm{t}(\mathrm{ms})$ & $\mathrm{CA}$ & PA & $\mathrm{v}\left({ }^{\prime \prime} / s\right)$ & $\phi(\mathrm{mas})$ \\
\hline SAO79031 & $\mathrm{R}$ & $641 \pm 58$ & 2 & $63 \mathrm{~S}$ & 122 & 0.35 & 1.6 \\
\hline
\end{tabular}

Table 1 shows ephemerides for the SAO79031 occultation, acquired using the scanning scheme described in Section 2.. The occultation was recorded storing a 20-pixel line every 2ms. Subsequent lightcurve shown in Figure 1 was calculated by averaging central pixels of every line and subtracting background estimated from the outer ones. A quick look reveals at least three diffraction fringes until signal vanishes into noise. This can be separated into two independent components, one due to residual periodic components in the CCD power supply and cooler and the other due to random atmospheric scintillation. While 
the first can be taken into account in the lightcurve fitting model, the latter lacks any deterministic approach. A detailed description of the adopted model, for fitting data to a theoretical occultation pattern, can be found in Richichi, Lisi, \& Di Giacomo (1992). A preliminary analysis has revealed that the SNR of the current lightcurve is insufficient to derive a confident stellar angular diameter from that model. Thus, we fit the lightcurve using a point source model, also taking into account both telescope and filter bandwidth smearing. As can be seen in Figure 1, the model +periodic noise curve is in good accordance with the data points. At the bottom, the residuals give some idea of the behaviour of the scintillation component of noise.

A more detailed assessment of limiting angular resolution of such technique will be presented in a foregoing paper (Fors \& Núñez 2001).

\section{Application of Fast Drift-Scanning to Speckle Interferometry}

Speckle interferometry (SI) and Speckle Imaging (Horch, 1995) provide diffraction limited resolution in small fields (isoplanatic patch) allowing, for example, to measure angular separations in close binaries far beyond the seeing. Thus, consecutive speckle campaigns allow us to trace the orbit of a close binary system. The technique consists of taking object images with a certain amount of time $\tau$ elapsing between them. This $\tau$ should be shorter than the speckle time, ranging from 20 to $40 \mathrm{~ms}$.

As in the case of lunar occultations, full frame CCDs are not fast enough for such $\tau$. However, modified drift-scanning technique enables these detectors to image at this rate. In particular, drift-scanning allows us to use an observing technique similar to the charge transfer used by Horch, Ninkov, \& Slawson (1997) but not limited in the strip size to the true size of the detector. This is because drift-scanning can be performed indefinitely. Thus, thousands of frames can be obtained continuously.

As a preliminary example we observed $\zeta$ Her $\left(2.9 \& 5.5 \mathrm{mag} ; 1.5^{\prime \prime}\right)$ on $07 / 21 / 2000$. The CCD scale was $0.3 /$ pixel, just roughly above the diffraction limit of the telescope. Effective exposure time was $40 \mathrm{~ms}$ which is right on the advisable limit for a $1.5^{\prime \prime}$ seeing night with reasonable wind conditions. Figure 2 shows ten of the obtained frames. Each frame is about $4 \times 4$ arcsecs. In Figure 2 is it easy to see that we have beat the $1.5^{\prime \prime}$ seeing, with both stars clearly separated. Although no detailed interferometric analysis has been carried out for angular separation, a quick visual inspection of frames shown in Figure 2 gives us $\rho=1.6^{\prime \prime}$ and $\alpha=90^{\circ}$. This result seems to be at least compatible with literature values: $\rho(1980)=1.3^{\prime \prime}, \alpha(1980)=140^{\circ} ; \rho(1990)=1.6^{\prime \prime}, \alpha(1990)=83^{\circ}$.

This result, although very preliminary, shows that it is possible to use the modified drift-scanning technique to perform speckle observations using common CCD cameras and small telescopes.

\section{The New Observatory Project}

Although this paper is primarily devoted to showing that the drift-scanning technique can be used to obtain millisecond photometric observations using small telescopes, given the characteristics of this meeting, it should be of some interest 


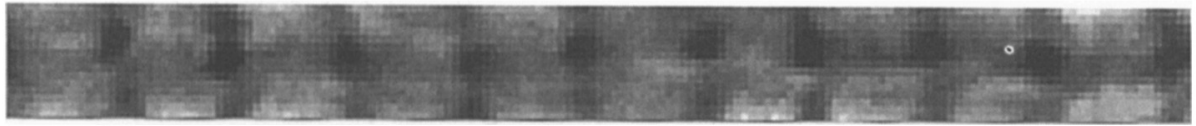

Figure 2. Speckle observation of $\zeta$ Her $\left(2.9 \& 5.5 ; 1.5^{\prime \prime}\right)$ at Fabra Observatory.

to present here some aspects of our project to build a new remotely controlled observatory near Barcelona.

The site of the new project, called Niu de l'Aguila, is at the top of one of the highest mountains $(2531 \mathrm{~m})$ in the Cadi-Moixeró Regional Park, located 100 $\mathrm{km}$ north of Barcelona. It preserves a remarkable dark sky and presents other attractive characteristics as the surrounding area is protected by law (natural park law); the road is opened during summer and there is a ski resort during winter, it has electricity, water services, and a wireless communications antenna. We have 5 years of meteorological data for the area and there is already a usable building with live-in guard. We expect to reach a limiting magnitude to about $V=21$ which is enough for the programs we intend to develop.

We plan to install two robotic telescopes at the new observatory. The first one will be a refurbished Baker-Nunn Camera which is presently at the Real Observatorio de la Armada (ROA) in San Fernando (Spain). This is a modified Schmidt type telescope designed for tracking Earth satellites which gives an impressive photographic field of view (FOV) of $5 \times 30$ degrees with images of less than 20 microns spot size across the entire FOV. In the first stage, a modification of the camera for CCD operation will easily give a FOV of $2 \times 2$ degs or larger with a $2 \mathrm{kx} 2 \mathrm{k}$ CCD. To obtain a FOV of $5 \times 5$ degs, useful for a CCD, would require some additional optical modifications as described in Carter et al. (1992). We plan to fully adapt this camera for robotic and remote-controlled operation via internet using the experience of the ROA in the robotization of the Carlsberg Meridian Circle in La Palma and the San Fernando Meridian Circle located presently at El Leoncito (Argentina). Our plans for the second telescope are less definite but we expect to install a new-brand $1 \mathrm{~m}$-class robotic telescope similar to the ones described in several papers of these proceedings. Both telescopes will operate complementarily, using the large FOV of the Baker-Nunn Camera mainly for discovering and the telescope with a larger collecting power mainly (but not only) for follow-up observations.

The programs we plan to carry out with both telescopes are: Near Earth Object (NEO) discovery and tracking; the same for the main belt, Trans-Neptunian asteroids and comets; finding extra-solar planets by precise photometry of transits; SN and novae discovery; location of GRBs; surveys for variable stars; normal (stare operation) and high speed (drift-scan) photometry; and any other programs suitable for this kind of small telescopes.

\section{Conclusions}

We show that modified drift-scanning allows most full frame CCDs to be used for FPO as LO and SI. The instrumental settings needed to do this are very simple, 
with few mechanical and optical complements. This will bring the opportunity to most professional and high-end amateur observatories to do FPO. We plan to continue these kinds of observations, among others, in the new observatory we are planning to build near Barcelona.

Acknowledgments. This work was supported in part by the DGICYT Ministerio de Ciencia y Tecnología (Spain) under grant no. BP97-0903. O. Fors is supported by a fellowship from DGESIC Ministerio de Educación, Cultura i Deportes (Spain), ref. AP97 38107939.

\section{References}

Carter, B.D., Ashley, M.C.B, Sun, Y-S. \& Storey, J.W.V. 1992, Proc. ASA, 10, 74 .

Fors O. \& Núñez J. 2001, in preparation.

Horch, E. 1995, Int. J. Im. Syst. and Tech. 6, 4, 401.

Horch, E., Ninkov, Z. \& Slawson, R.W. 1997, AJ 114, 5, 2117.

Kristian J. \& Blouke M. 1982, Scientific American 247, 4, 48.

Ragazzoni R., Baruffolo A., Farinato J. 1998, Ghedina A., Mallucci S., Marchetti E. \& Niero T., 1998, SPIE 3353, 132.

Richichi A., Lisi F. \& Di Giacomo A. 1992, A\&A 254, 149.

Sabbey C.N., Coppi P. \& Oemler A. 1998, PASP 110, 1067.

Stone R.C., Monet D.G., Monet A.K.B., Walker, R.L. \& Ables, H.D. 1996, AJ $111,4,1721$. 